\title{
The Effect of Polymer Blend on the Third Order Nonlinearity of Oxazine Dye Doped Films by Using Z-Scan Techniques
}

\author{
Amal F. Jaffar \\ Ministry of High Education \& Scientific Researches, Middle Technical University, \\ Institute of Medical Technology, Baghdad-Iraq. \\ E-Mail: amaljaffar@ymail.com.
}

\begin{abstract}
The nonlinear optical properties of the laser dye Oxazine doped with polystyrene (OX /PS), Ox/poly methylmethacrylate (PMMA) and Ox/PS-PMMA blend films have been studded. The films deposited by free-casting technique and characterized by $\mathrm{Z}$ - scan technique .Z-scan experiment was performed using continuous wave $(\mathrm{CW})$ diode laser at $650 \mathrm{~nm}$ at in two parts. The first part was done using a closed-aperture placed in front of the detector to measure the nonlinear refractive index ,with the second part; the aperture in front the detector was removed (open aperture) to measure the nonlinear absorption coefficient. The open-aperture curve exhibits a normalized transmittance (valley), indicating the presence of induced absorption. The observed nonlinearity is found to be of the third order, as it fits to two photon absorption process (TPA). The closed-aperture curve exhibits a peak to valley shape, indicating a negative value of the nonlinear refractive index $\mathrm{n}_{2}$. The effective values of the nonlinear refractive index $\mathrm{n}_{2}$, the nonlinear absorption coefficient $\beta_{2}$, real and imaginary parts of the third-order optical nonlinearity, $\chi^{(3)}$ for Ox/PMMA-PS films comparatively with that of Ox/PMMA and Ox/PS has much lower transmittance value obtained shows that these films can be used as efficient optical limiters.
\end{abstract}

Keyword: Z-scan technique, nonlinear refraction and nonlinear absorption, dye doped polymer films, polymer blend.

\section{Introduction}

Nonlinear optical materials are required in a wide range of important applications, such as optical limiting, optical computing, and optical communication. [1]. With growing advancement in the technological field of photincs and bio-photincs, the search for organic materials exhibiting large nonlinear optical (NLO) properties and having applications in low power nonlinear optical devices has picked-up. Organic dyes are the most attractive optical materials for studying nonlinear optical properties for the point of view understanding their photo-physics and impending applications [2]. Dye chromophores are a class of organic molecules with multiple $\pi$-conjugated bonds, which can exhibit large optical nonlinearities and fast response time, as a result of the ease of polarization of their extended mobile $\pi$-electron clouds over large molecular distances. Strong absorption of dyes in the visible region makes them particularly suited for nonlinear optical investigations. It has also been shown that embedding dye chromophores in suitable host matrices enhances the lifetime and stability of the dyes entrapped within it [3].

The use of a synthetic polymer host presents advantages as these materials show much better compatibility with organic laser dyes and are amenable to inexpensive fabrication techniques. These polymers provide an opportunity for the production of active elements that can effectively control the characteristics of laser radiation. [4]. Polymer blends have become very important subject for scientific investigation in recent years because of their growing commercial acceptance, and they can possess unusual combinations of properties [5,6]. Copolymerization and blending are alternative routes for modifications of properties of polymers. Blending is the less expensive method, but polymer blends have been successfully used in an increasing number of applications in recent years. Such success encourages more attempts to apply this technique to a wider range of problems in polymer related industries [6]. The design of polymer blends constitutes an interesting alternative to obtaining micro-and 
nanostructured surfaces. The cost is reasonable and it is free from time-consuming procedures. Blending of polymers can yield materials with unprecedented properties that cannot be provided otherwise by using a single polymer. [7,8]. Polystyrene-an amorphous, optically clear thermoplastic material, which is flexible as thin films, is chosen as the host matrix because of its ideal properties for investigating the optical properties of the Ox/PS films. Polystyrene (PS) is in solid (glassy) state at room temperature but flows if heated above its glass transition temperature and becomes solid again when cooled [9]. Polymethylmethacrylate is an amorphous thermoplastic that can be delivered as clear as glass. The material is more known as "Plexiglass". PMMA is produced by the addition polymerization of methylmethacrylate. The polymer has very good optical properties but has poor scratch resistance. It has good dimensional stability due to rigid polymer chains. It has good weather resistance, and is stable to acid and alkalis. It is attacked by several organic solvents, and has good impact strength higher than that of glass or polystyrene. It has the best transparency and optical properties of commercially available thermoplastic. It is colorless with a $92 \%$ light transmission, an index of refraction of 1.49. It comes in a full range of transparencies [9].

The basic absorption processes in dyes can be divided into linear and nonlinear absorption. Nonlinear optics is a material phenomenon in which intense light induces a nonlinear response in the medium, and in return the medium modifies the optical fields in a nonlinear way. In fact, all media are nonlinear to a certain degree [10].

A nonlinear absorber of light has the unique property that its optical absorption can be altered by introducing changes in the intensity of radiation incident upon it. [11,12].

$\mathrm{Z}$ scan technique is a simple, sensitive and popular experimental method to measure nonlinear optical prosperities (NLO) of materials. There were two parts of the Z-scan: closed aperture and open aperture. Closed aperture Z-scan helps to measure the sign and magnitude of real part of third order (NLO) and nonlinear refractive index $\left(\mathrm{n}_{2}\right)$.Open aperture Z-scan either two types: saturable absorption (SA) and reverse saturable absorption (RSA) depending on the pump intensity and on the absorption cross section at the excitation wavelength [11]. Open aperture Z-scan helps to measure the nonlinear absorption coefficient $\beta_{2}$ and the imagenary part of of third order (NLO) [9].

In the present work, the samples films of oxazine be solved in Tetrahydrofloran (THF) and the host matrix used are PMMA, PS and the blend of the two polymers, PS and PMMA have been synthesized and their linear and nonlinear optical properties, investigated. Both of these polymers are highly transparent and environmentally stable and proven to be excellent for forming doped films with Oxazine with high nonlinear proprieties.

There are no detailed studies on the nonlinear optical characterization of dye/polymer bleding films. The work presented in this paper is an attempt to investigate in detail the nonlinear optical properties of the free casting Ox/PS-PMMA films and see how the choice of the polymer blend is influencing the linear and nonlinear optical characteristics. The results obtained are compared with those of the Ox/PS and Ox/PMMA composite films.

\section{2-Theory}

\section{2-1 Closed - Aperture Z- Scan:}

The standard "closed aperture" Z-scan (i.e. aperture is placed in the far field) for determining nonlinear refraction is shown in Fig.(1), where the sample is moved along the propagation direction $\mathrm{z}$ while keeping the input pulse energy fixed. The normalized transmittance of the sample through the aperture is monitored in the far field as a function of the position $\mathrm{Z}$. Intensity dependent on the refractive index causes the beam radius of the transmitted beam to change while retaining the Gaussian profile.

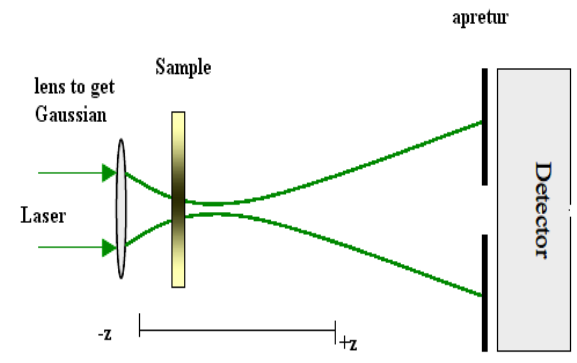

Fig.(1) The scheme of the closed aperture $z$-scan [13]. 
The size of the aperture is signified by its transmittance $(S)$, in the linear regime. In most reported experiments, $0.1<\mathrm{S}<0.5$ has been used for determining nonlinear refraction. [14].

The Z-scan technique is based on the transformation of phase distortions to amplitude distortions during beam propagation. A qualitative physical argument that explains the transmittance variations in the Z-scan experiment can be given as follows: Starting the scan from a distance far away from the focus (negative $\mathrm{z}$ ), the beam irradiance is low and negligible nonlinear refraction occurs leading to linear transmittance. As the sample is brought closer to the focus, the beam irradiance increases leading to self-lensing in the sample. A negative self-lensing (self-defocusing) prior to focus tends to collimate the beam and reduce the diffraction leading to a smaller beam at the aperture and an increased transmittance. As the scan continues and the sample crosses the focal plane to the right (positive $\mathrm{z}$ ), the same self-defocusing effect will tend to augment diffraction and reduce the aperture transmittance. A prefocal transmittance maximum (peak) and a post focal transmittance minimum (valley) will be, the $\mathrm{z}$ scan signature of a negative nonlinearity as shown by the dote line Fig.(2), while a positive one, following the same analogy, will give rise to an opposite valley-peak configuration, as shown by Solid line Fig.(2). [15].

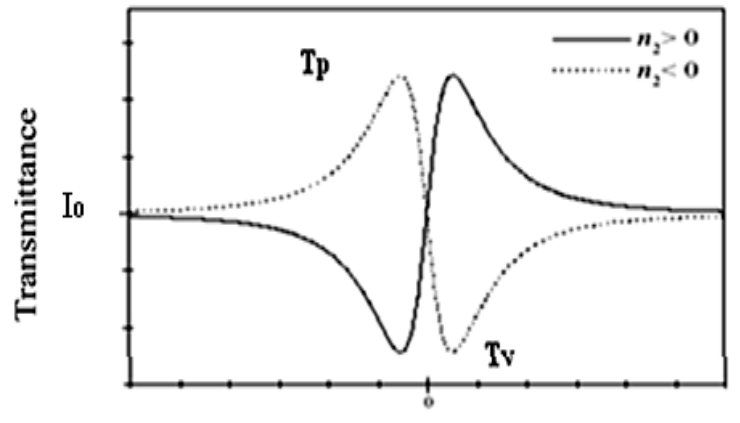

position (z)

Fig.(2) Calculated Z-scan transmittance curves for a third order nonlinearity [14].

\section{2-2 Self Focusing and Defocusing:}

Self-focusing (or Kerr-Lansing) is a consequence of the non-uniform spatial profile of the laser Gaussian beam. If the intensity of a transmitted light beam is sufficiently high, the refractive index change will modify the light propagation not only with respect to the polarization but in its geometrical properties too [16].

For a Gaussian beam of radius $\omega_{0}$ (beam waist) the Kerr-lens focal length is:

$\mathrm{F}=\mathrm{a} \omega_{0}^{2} / 4 \operatorname{Ln}_{2} \mathrm{I}$.

Where $\mathrm{L}$ is the thickness of the nonlinear medium (sample), (I) is the irradiance and (a) is a correction term.

When $\mathrm{n}_{2}$ is negative, the above equation shows there will be a negative focal length and thus self de-focusing of the incident beam [14].

The variation in refractive index will produce a phase shift in the pulse, leading to a change of the pulse's frequency spectrum. A pulse (top curve, Fig. (3)) propagating through a nonlinear medium undergoes a selffrequency shift (bottom curve) due to selfphase modulation. The front of the pulse is shifted to lower frequencies, the back of the pulse to higher frequencies. In the centre of the pulse the frequency shifted is approximately linear $[17,18]$.

If the intensity of a transmitted light beam is sufficiently high almost every material, gases, liquids or solids, will show a nonlinear interaction. Thus the refractive index will be changed as:

$\Delta \mathrm{n}_{\mathrm{x}}=\mathrm{K}_{\text {kerr }} \lambda_{\text {inc. }}\left(3 / 4 \mathrm{E}^{2}\right.$ inc., $\left.\mathrm{x}+\mathrm{E}_{\text {ext }}^{2}\right)$

Where $K_{\text {kerr }}$ is Kerr constant, $\lambda$ inc.: incident wave length, $\mathrm{E}$ inc. the incident electric field, $E_{\text {ext }}$ is the external electric field, if the nonlinear range of the electric field or intensity is reached. In particular, if light beams with transverse intensity profile, as Gaussian beams, are applied this refractive index change will be different over the cross-section of the beams [14].

As a consequence for high-intensity beams with long interaction lengths in the matter selffocusing can occur and for short interaction lengths self-defocusing may be obtained [14].

We define the change in transmittance between the peak and valley in a Z-scan as:

$\Delta \mathrm{T}_{\mathrm{pv}}=\mathrm{T}_{\mathrm{p}}-\mathrm{T}_{\mathrm{V}}$ 
where $T_{p}$ and $T_{v}$ are the normalized peak and valley transmittances as seen in Fig.(2) The empirically determined relation between the induced on axis phase shift, $\Delta \Phi_{0}$, and $\Delta \mathrm{T}_{\mathrm{pv}}$ for a third-order nonlinear refractive process in the absence of nonlinear absorption (NLA) is,

$$
\Delta T_{p v \cong} \cong 0.406(1-s)^{0.27}\left|\Delta \phi_{o}\right|
$$

S: the size of the aperture.

If the Z-scan aperture is closed to allow linear transmission of less than 10 percent or $0.1<\mathrm{S}<0.5$, then:

$$
\Delta T_{p v} \cong 0.406\left|\Delta \phi_{o}\right|
$$

The nonlinear refractive index is calculated from the peak to valley difference of the normalized transmittance by the following formula: -

$$
\begin{aligned}
& \mathrm{n}_{2}=\Delta \phi_{o} / \mathrm{I}_{\mathrm{o}} \mathrm{L}_{\mathrm{eff}} \mathrm{k} \\
& \mathrm{Io}={ }^{2} P_{\text {peak }} / \pi \omega_{o}^{2}
\end{aligned}
$$

$I_{o}$ is on the axis irradiance,

$\omega_{o}$ : The beam radius at the focal point, $\lambda$, is the wavelength of the beam.

$L_{\text {eff }}$ : The effective length of the sample (thickness), can determined from the following formula:

$$
L_{\text {eff }}=\left(1-e^{-\alpha_{o}} L\right) / \alpha_{o}
$$

Where,

L: the sample thickness

$$
\begin{aligned}
& \alpha_{o}: \text { Linear absorption coefficient, } \\
& \alpha_{o}=\frac{1}{L \ln \left(\frac{1}{T}\right)} \ldots \ldots \ldots \ldots \ldots \ldots \ldots \ldots \ldots \ldots \ldots \ldots \ldots \ldots \ldots \ldots \ldots \ldots \ldots
\end{aligned}
$$

where T: linear transmittance [19] .

\section{2-3 Open Aperture Z-Scan:}

An open-aperture Z-Scan measures the change in intensity of a beam, focused by lens $\mathbf{I}$ in Fig.(3), in the far field at detector PD, which captures the entire beam, and gives the estimate of the absorptive nonlinearity of a sample [20]. The change in intensity is caused by multi-photon absorption in the sample as it travels through the beam waist. In the focal plane where the intensity is greatest, the largest nonlinear absorption is observed. At the "tails" of the Z-scan signature, where $|Z| \gg Z_{0}$, the beam intensity is too weak to elicit nonlinear effects. The higher order of multi-photon absorption present in the measurement depends on the wavelength of light and the energy levels of the sample [19].

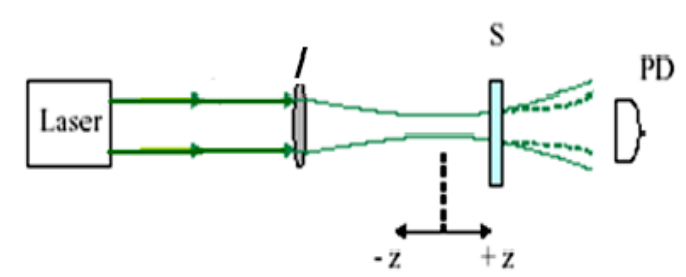

Fig.(3) Open-aperture Z-Scan [13].

The absorptive nonlinearity can be due to either (i) saturable absorption (SA), in which the absorption coefficient decreases resulting in the transmittance increase with increase in the input laser intensity, and (ii) reverse saturable absorption (RSA), in which the absorption coefficient increases resulting in the transmittance decrease with increase in the input laser intensity [16]. The normalized change in transmitted intensity can be approximated by the following equation, [10, and 16]:

$T(z)=\sum_{m=0}^{\infty} \frac{\left[\frac{\beta I_{o} L_{e f f}}{1+(Z / Z o)^{2}}\right]^{m}}{(m+1)^{3 / 2}}$

where,

$\mathrm{Z}$ : is the sample position at the minimum transmittance, $m$ : integer,

$\mathrm{Z}_{0}$ : Rayleigh range

$\mathrm{Z}_{0}={\mathrm{n} \pi \mathrm{w}_{0}}^{2} / \lambda$

$\mathrm{T}(\mathrm{z})$ : the minimum transmittance,

Experimentally determined nonlinear refractive index $\mathrm{n}_{2}$ and nonlinear absorption coefficient $\beta$ can be used in finding the real and imaginary parts of the third-order nonlinear optical susceptibility $\left[\chi^{3}\right]$ according to the following: [21] 
$\operatorname{Re} \chi^{(3)}(\mathrm{esu})=10^{-4} \varepsilon_{0} c^{2} n_{0}{ }^{2} n_{2} / \pi\left(\mathrm{cm}^{2} / \mathrm{W}\right)$

$\operatorname{Im} \chi^{(3)}(\mathrm{esu})=10^{-2} \varepsilon_{0} c^{2} n_{0}{ }^{2} \lambda \beta / 4 \pi^{2}(\mathrm{~cm} / \mathrm{W})$

Where $\varepsilon_{0}$ is the vacuum permittivity and $c$ is the light velocity in vacuum. [22]

The absolute value of the third-order nonlinear optical susceptibility is given by the relation:

$\mid \chi^{3} \|=\left[\left(\operatorname{Re}\left(\chi^{3}\right)\right)^{2}+\left(\operatorname{Im}\left(\chi^{3}\right)\right)^{2}\right]^{1 / 2}$

\section{Experimental Section:}

Oxazine is from xanthene family with fluorescence emission in the yellow -red region of the electromagnetic spectrum are well known for their excellent laser performance in liquid solutions as well as in solid matrices [23], Fig.(4) Shows its molecular structure.

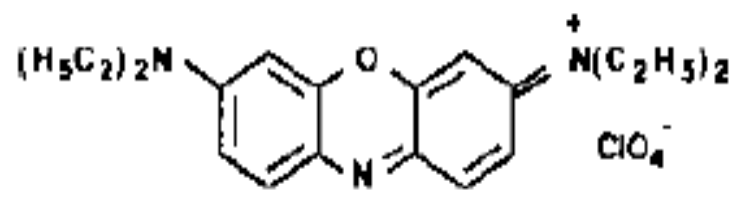

Fig.(4) The molecular structure of Oxazine, [23].

Polymethylmethacrylate (PMMA) and Polystyrene (PS) are from ICI Company, were reported to have molecular weights of 145,000 and $167,000 \mathrm{~g} \cdot \mathrm{mol}^{-1}$, respectively. Tetrahydrofloran (THF): from (BDH) laboratory reagents (BDH Chemicals Ltd Poole England). Moreover, Oxazine obtained from Lambdacxhrome. Laser Dye Oxazine was used in this study without further purification.

The thicknesses of the films were measured with an electrical device (Minitest 3000 microprocessor coating thickness) from electro, phyisk, Germany (ERICHSEN). The films had thickness of (1.72-1.87 $\mu \mathrm{m}$. Visible Shimadzu spectrophotometer (UV 160), which operates in wavelength range of $200 \mathrm{~nm}$ to $1100 \mathrm{~nm}$ and with scanning speed of $1500(\mathrm{~nm} / \mathrm{min})$, carried out UV-Visible spectroscopy measurements. The $Z$-scan experiments were performed using $650 \mathrm{~nm} \mathrm{CW}$ diode laser, (max.power is
$50 \mathrm{~mW}$, Ac: 220-240 volt, Frequency: 50-60 Hz 250mA.beam diameter: $1.5 \mathrm{~mm}$, beam divergent $1.5 \mathrm{mrad}$, which was focused by $10 \mathrm{~cm}$ focal length lens. The laser beam waist $\omega_{0}$ at the focus is measured to be $0.015 \mathrm{~mm}$ and the Rayleigh length $\mathrm{Z}_{0}$ to be $0.92 \mathrm{~cm}$.

\section{Samples preparation}

Oxazine solution of concentrations $\left(10^{-5}\right) \mathrm{M}$ in THF is prepared by weighting amount of the material by using a matter balance having a sensitivity of $10^{-4} \mathrm{gm}$. PMMA, PS, and Ox had been dissolved in THF separately. Ox was added to the two homopolymers and also their blend (PS/PMMA 50/50). The doping ratio was $1: 2$ dyes to the polymer.

Dye doped polymer films were fabricated by the free casting technique (FC). The free casting (FC), involves casting a polymer solution in a flat bottomed glass cup (Petri dish) without imposing hydrodynamic stress on the liquid. The dishes were arranged on a glass plate in order the dishes have a plan situation, and then the dishes were covered with a heavy paper box to be protect from light and dust. Solvent is allowed to evaporate under ambient conditions $\left(30 c^{\mathrm{o}}\right)$ until the films hardens. The harden films were then removed from the Petri dish by washing it off with distilled water and placed in the oven $\left(50 \mathrm{c}^{\mathrm{o}}\right)$ for 10 minutes to dry .

\section{Results and Discussion}

UV-Visible absorption spectra of the laser dye Oxazine and its doped polymer films were carried out by using (UV/Vis SP - 3000, plus, 2003) Spectrophotometer Optima/Japan, which operates in wavelength range of 190$1100 \mathrm{~nm}$ [24].

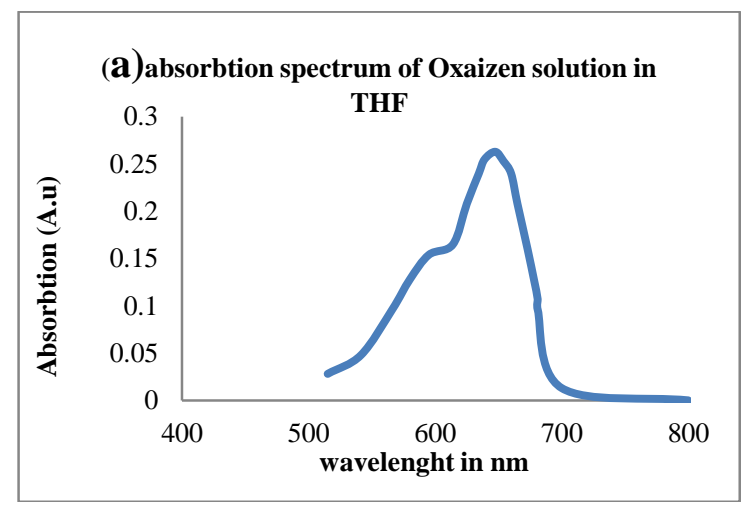

Fig.(5) Linear absorption spectra of Ox solution. 


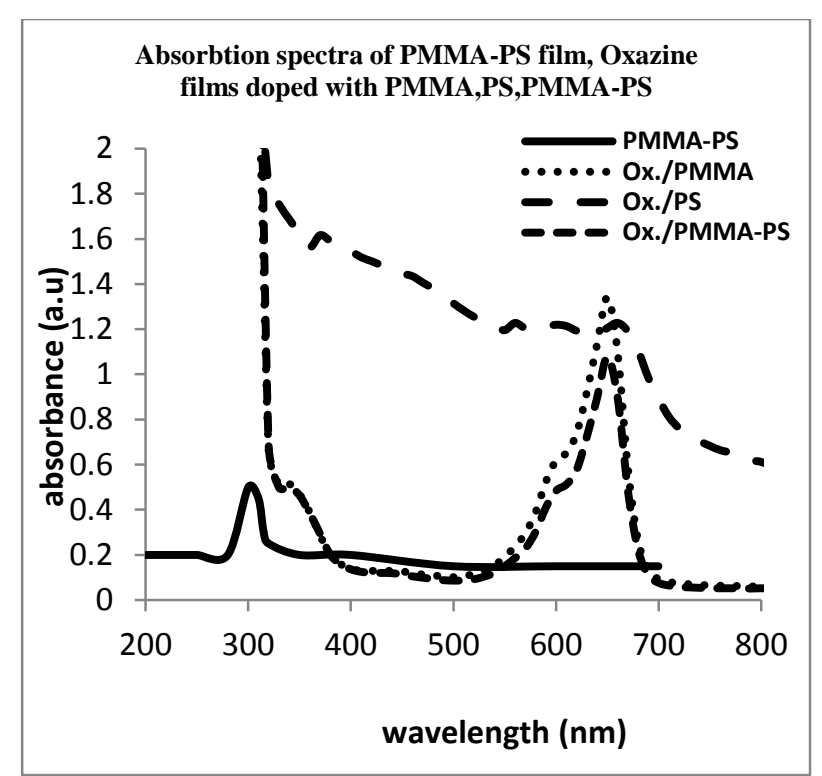

Fig.(6) Linear absorption spectra of $O x$ dye doped films.

Fig. (5) shows the absorption spectrum at room temperature for Oxazine solution $10^{-5} \mathrm{M}$ in THF. Fig.(6) shows absorbtion spectra of Pure PS-PMMA blend films, oxazine / PMMA films, Oxazine/ PS films, and their blend (PMMA/PS 50/50) measured in a range of wavelengths from 200 to $800 \mathrm{~nm}$. Pure PS-PMMA blend films do not show any appreciable UV absorption, but an absorption window is found in the range of 250-365 nm. For the Ox. /PMMA-PS composite films exhibit excellent UV absorption window of about $200 \mathrm{~nm}$ width. Furthermore, the absorption peak wavelength of the PMMA-PS films is substantially blue shifted relative to that of Ox./PMMA-PS films $(\sim 350 \mathrm{~nm})$ due to the strong confinement effect [25]. The presence of Oxazine, thus, enhances the UV absorption of the composite films and modifies the overall optical behaviour of polymer blend films [26].

Also, it can be seen that the absorption peak value for Ox. / PMMA was greater than that in Ox./PMMA-PS 50/50), which were also greater than that in Ox./PS, for the same concentrations of the doped dye.

Nonlinear optical properties (the third-order nonlinear refractive index $\mathrm{n}_{2}$ and the nonlinear absorption coefficient $\beta$, third order susceptibility (3) of Oxazine doped films with PMMA, PS and their blend were investigated by Z-scan technique by using $\mathrm{cw}$ diode laser with $650 \mathrm{~nm}$ and incident intensity
$\mathrm{I}_{0}=509.6 \mathrm{~W} / \mathrm{cm}^{2}$. Fig.(5) shows the typical open aperture Z-scan profiles of the films. When the sample is away from the focus $Z=0$, the incident laser intensity is low and linear so it is normalized to unity. When it moves close to the focus, the OA Z-scans exhibits a reduction in the transmission which is symmetric about the focus $\mathrm{Z}=0$. This is typical of an intensity dependent enhanced absorption termed reverse saturable absorption (RSA) or positive nonlinear absorption exhibited by a nonlinear material and can be exploited for protection of eyes and sensors against radiation induced damage. The observed nonlinearity is found to be of the third order, as it fits to a two photon absorption process (TPA). From the open aperture Z-scan curve it is found that, the ox/PS-PMMA films do exhibit large induced absorption behaviour. The observed dip in the open aperture curve shows the transmittance limiting efficiency of the films. The transmittance minimum obtained for Ox/PS film is about 0.192, Ox/PMMA film is about 0.089 and the curve of Ox/PS-PMMA films show a better fit to the theoretical equations for TPA, and the“ transmittance minimum is about 0.026 , which highlights the better optical limiting efficiency of the latter compared to the former ones. The calculated nonlinear parameters are given in Table (1). The comparatively much lower transmittance value obtained for Ox/PS-PMMA composite films Fig.(7) shows that these films can be used as efficient optical limiters. Optical limiting (OL) devices protect light-sensitive sensors, such as eye or CCD cameras, from possible damage caused by intense light exposure. The observed improvements in the nonlinear optical properties of Ox/PS-PMMA films compared to Ox/PS and Ox/PMMA films can be associated with the formation of an interpenetrating network (IPN) of PS and PMMA in the PS-PMMA polymer blend. 


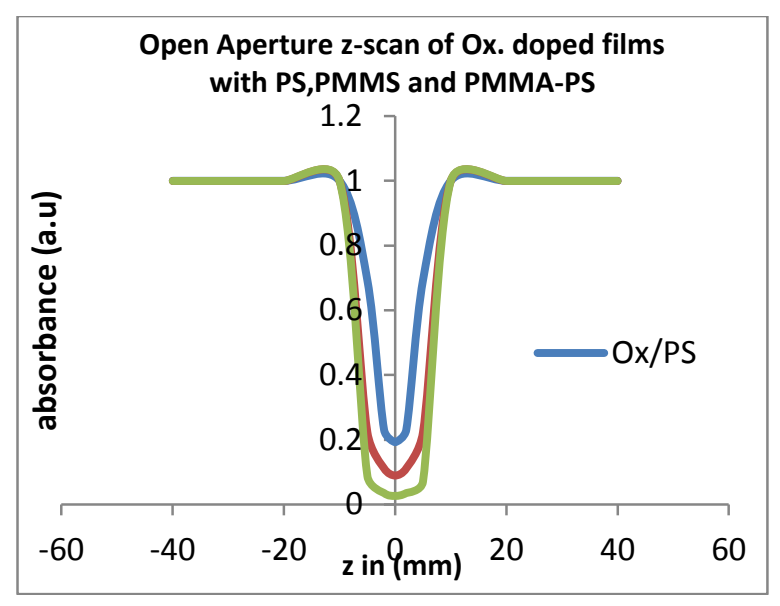

Fig.(7) OA Z-scan profile of oxazine doped films in PMMA,PS and their blend $t$ $I_{0}=509.6 \mathrm{watt} / \mathrm{cm}^{2}$.

Table (1)

Nonlinear parameters of Ox./PMMA, Ox./PS, Ox./PMMA-PS films by using $C W$ diode laser at $650 \mathrm{~nm}$ for open aperture z-scan.

\begin{tabular}{||c||c||c||c||c|}
\hline films & $\boldsymbol{T}(z)$ & $\begin{array}{c}\boldsymbol{\beta} \\
(\mathbf{c m} / \text { watt })\end{array}$ & $\boldsymbol{I m} \chi^{3}(\mathbf{e s u})$ & $\begin{array}{c}\chi^{3} \\
(\boldsymbol{e s u})\end{array}$ \\
\hline \hline Ox./PMMA & 0.070 & 0.034 & $1.09 \mathrm{E}+00$ & 1.085 \\
\hline \hline \begin{tabular}{c} 
Ox./PS \\
\hline $\begin{array}{c}\text { Ox./PMMA- } \\
\text { PS }\end{array}$
\end{tabular} & 0.089 & 0.206 & $2.52 \mathrm{E}+00$ & 2.516 \\
\hline
\end{tabular}

Closed aperture Z-scan profile (The defocusing effect) of Oxazine doped films in PMMA, PS and their blend for $\mathrm{Io}=509.6 \mathrm{GW} / \mathrm{cm}^{2}$ are shown in Fig.(8). Fig (8) indicates to negative refractive index is attributed to a thermal nonlinearity resulting from the absorption of radiation at $650 \mathrm{~nm}$. Analogy to the theoretical behavior of the dotted line in Fig.(2).

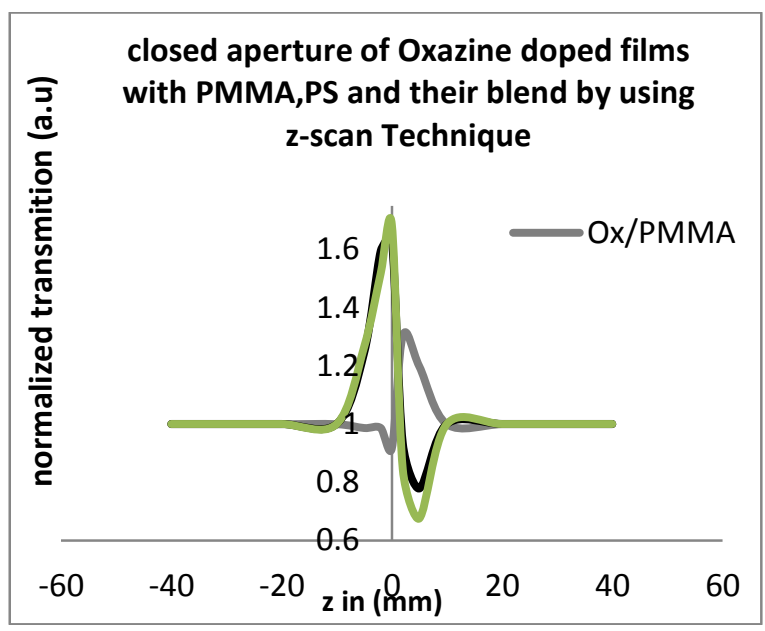

Fig. (8) CA Z-scan profile of Oxazine doped films for $I o=509.6 \mathrm{GW} / \mathrm{cm}^{2}$ indicates to negative.

Refractive index for the three films (Ox /PMMA , Ox /PS, Ox PMMA-PS).

The experimentally determined values of $T \mathrm{p}-\mathrm{v}, \mathrm{n}_{0}, n_{2}, \Delta \phi o$ and $\mathrm{R} \chi 3$ are given in Table (2). The experimentally determined values of $\beta, \operatorname{Im} \chi^{3}, \chi^{3}$ are given in Table (1).

Table (2) shows that Ox./PMMA-PS film has the highest nonlinear parameter compared with Ox./PMMA and Ox./PS film.

Table (2)

Nonlinear parameters of Ox./PMMA, Ox./PS,Ox./PMMA-PS films by using CW diode laser at $650 \mathrm{~nm}$ for open aperture $z$-scan.

\begin{tabular}{|c|c|c||c||c||c||c||c||}
\hline films & $\boldsymbol{T p}-\boldsymbol{v}$ & $\boldsymbol{n}_{\boldsymbol{o}}$ & $\boldsymbol{n}_{2}\left[\mathbf{c m}^{2} / \boldsymbol{W}\right]$ & $\boldsymbol{\Delta \phi o}$ & $\boldsymbol{R} \boldsymbol{\chi}^{3} \boldsymbol{e s u}$ & $\boldsymbol{I m} \boldsymbol{\chi}^{3}(\boldsymbol{e s u})$ & $\begin{array}{c}\chi 3 \\
(\boldsymbol{e s u})\end{array}$ \\
\hline \hline Ox./PMMA & 0.388 & 2.738 & $2.568 \mathrm{E}-06$ & 0.955 & $1.256 \mathrm{E}-05$ & $1.09 \mathrm{E}+00$ & 1.085 \\
\hline \hline Ox./PS & 0.856 & 1.656 & $5.322 \mathrm{E}-06$ & 2.108 & $1.972 \mathrm{E}-05$ & $2.52 \mathrm{E}+00$ & 2.516 \\
\hline \hline Ox./PMMA-PS & 1.013 & 3.448 & $7.858 \mathrm{E}-06$ & 2.494 & $1.86 \mathrm{E}-04$ & $4.60 \mathrm{E}+00$ & 4.599 \\
\hline
\end{tabular}

With cw pumping we expect major contribution to the observed third-order nonlinearities to be thermal in nature. The energy from the focused laser beam is transferred to sample through linear absorption and is manifested in terms of heating the medium leading to a temperature gradient and there by the refractive index change across the sample which then acts as a lens. The phase of the propagating beam will be distorted due to the presence of this thermal lens. The peak-valley separation of more than 1.7 times the Rayleigh range of $\sim 10.8 \mathrm{~mm}$ also suggests the presence of thermal component in our case. 
It is well established that a separation of $\sim 1.7 \mathrm{z}_{0}$ indicates Kerr-type of nonlinearity [7].

The observed higher nonlinear susceptibility of Ox/PS-PMMA films measured by the Z-scan technique, compared to both Ox/PS and Ox/PMMA films, establishes the fact that the Ox/PS-PMMA films have better nonlinear optical response and can be chosen as ideal candidates with high prospects of applications in nonlinear optics [27].

There are no detailed studies on the nonlinear optical characterization of Ox/PSPMMA films or other kinds of dyes, but comparing with ZnO/PMMA-PS films [27], $\left(\chi^{3}\right)$ of Ox/PMMA-PS films is the highest and the transmittance minimum of the two photon absorption curve is the lowest which highlights the better optical limiting efficiency of the latter compared to the former one.

\section{Conclusion}

Nonlinear optical properties (the third-order nonlinear refractive index $\mathrm{n}_{2}$, the nonlinear absorption coefficient $\beta_{2}$, and third order susceptibility $\chi(3)$ of Oxazine doped films with PMMA, PS and their blend were investigated by $\mathrm{Z}$-scan technique by using $\mathrm{cw}$ diode laser at $650 \mathrm{~nm}$ and incident intensity $\mathrm{I}_{0}=509.6 \mathrm{~W} / \mathrm{cm}^{2}$. From the open aperture Z-scan curve the observed nonlinearity for the three films Ox/PMMA,OX/PS and Ox/PMMA-PS is found to be of the third order, as it fits to a two photon absorption process (TPA). It is found that, the ox/PSPMMA films do exhibit large induced absorption behaviour. The observed dip in the open aperture curve shows the transmittance limiting efficiency of the films which highlights the better optical limiting efficiency compared to Ox/PMMA and Ox/PS. The observed improvements in the nonlinear optical properties of Ox/PS-PMMA films compared to Ox/PS and Ox/PMMA films can be associated with the formation of an interpenetrating network (IPN) of PS and PMMA in the PS-PMMA polymer blend. Closed aperture $\mathrm{Z}$-scan indicates to negative refractive index is attributed to a thermal nonlinearity resulting from the absorption of radiation at $650 \mathrm{~nm}$. The observed higher nonlinear susceptibility of Ox/PS-PMMA films (Higher $\mathrm{n}_{2}$ and $\beta_{2}$ ) measured by the $\mathrm{Z}$-scan technique, compared to both $\mathrm{Ox} / \mathrm{PS}$ and Ox/PMMA films, establishes the fact that the Ox/PS-PMMA films have better nonlinear optical response and can be chosen as ideal candidates with high prospects of applications in nonlinear optics.

\section{References}

[1] Ahmad Y. Nooraldeen, Palanichant M., Palanisamy P. K., "Influence of Solvents Polarity on NLO Properties of Fluorone Dye", International Journal of Nonlinear Science 7(3), 290-300, 2009.

[2] Sana Z, Zahid H. Khan, Mohd. Shahid Khan, "Experimental and theoretical investigations of nonlinear optical properties of 1,4- Diamino-9, 10-Anthraquionone, Spectrochimica Acta Part A: Molecular and Biomolecular Spectroscopy", 114, 164-169, 2013.

[3] Rekha R. K., Ramalingam A, "Nonlinear characterization of Mercurochrome dye for potential application in optical limiting", XL, 1, 187-196, 2010.

[4] Achamma K., Nibu A. G., Binoy P., P., Nampoori V. P. N. and Vallabhan C. P. G., "Fluorescence Efficiency and Photodegradation of Rhodamine 6G Doped PMMA Using a Dual Beam Thermal Lens", Technique Laser Chemistry" 20(2-4), 99110, 2002.

[5] Zheng. S, Huang. J, Li.J, Guo.Q, "Phase Behavior and Properties of Poly (methylmethacrylate)/ Poly (vinyl acetate) Blends Properties Via in Situ Polymerization", J.of Applied Polymer Science,.69, 4 , 675-684,1998.

[6] Bhatangar M.S., "Textbook of Polymers: Processing and Applications" Company LTD New Delhi 2, 2004.

[7] Joel, R. F., "Polymer Science \& Technology", Prentice-Hall, Inc, $2^{\text {nd }} \mathrm{Ed}$, (2005).

[8] Juan R.-H., "Nano/Micro and Hierarchical Structured Surfaces in Polymer Blends, Nanostructured Polymer Blends, 357-421, 2014.

[9] Marwa R. F., "Study of Thermal Aging Effect on Optical Properties of Some Polymer Blends", Msc thesis, 2010. 
[10] Sheik-Bahae M., Said A.A., Wei T.H., Hagan D.J. and Van Stryland E.W., "Sensitive Measurement of Optical Nonlinearities Using a Single Beam" IEEE Journal of Quantum Electronics, QE-26, 760-769, 1989.

[11] Menze R., "Photonics", Springer, Berlin, 2001.

[12] Steven R. V., "Nonlinear-Optical Studies Of Organic Liquids And Polymer Optical Fibres", phd Thesis, Washington State University, Department of Physics, 2000.

[13] Sheik-Bahae M., Said A.A., Wei T.H., Hagan D.J. and Van Stryland E.W., IEEE Journal of Quantum electronics. 26, 760769, 1990.

[14] Sheik- Bahae Mr, Michael P H., "Handbook of Optics,. IV, 17, 2000.

[15] Kaladevi S., Vijayan C.,Kothiyal M. P., "Low-threshold optical power limiting of $\mathrm{cw}$ laser illumination based on nonlinear refraction in zinc tetraphenyl porphyrin", Optics \& Laser Technology, 38, 512-515, 2006.

[16] Mansoor S. B., "Characterization Techniques and Tabulations for Organic Nonlinear Materials", Inc, 1998.

[17] Geata A. L. "Physical Review Letter", 84, 3582, 2000.

[18] Moll K. D. "Physical Reviow Letter", 90, 203902, 2003.

[19] Mansoor S. B, Said Au A., Hagan D. J., Soileau M. J., Nonlinear refraction and optical limiting in thick media "Optical Engineering”, Vol.30, No. 8, pp 1228$1235,1991$.

[20] Sailaja R., PhD thesis, Indian Institute of Technology Madras Chennai India, 2007.

[21] Mansoor S. B, David J. Hagan, "Sensitive Measurement of Optical Nonlinearities, Using a Single Beam", IEEE LEOS NEWSLETTER. 17-26, 2007.

[22] Mathews S.J, Chaitanya Kumar S., Giribabu L., Venugopal Rao S., "Nonlinear optical and optical limiting properties of phthalocyanines in solution and thin films of PMMA at $633 \mathrm{~nm}$ studied using a $\mathrm{cw}$ laser", Materials Lett. 61, 4426-4431, 2007.

[23] Brackmann U., "Lambdacxhrome Laser Dyes", Lambda Physik ,GmbH,1986.
[24] Manual of SP_3000 Puls $\mathrm{Uv} / \mathrm{Vis}$ Spectrophotometer Optima /Japan.

[25] Haase M; H Weller and A Henglein, J. Phys. Chem. 92, 482, 1988.

[26] Ahmed R. M., and Saif M., Optical Properties of Rhodamine B Dye Doped in Transparent Polymers for Sensor Application, Chinese Journal Of Physics. 51,. 32013.

[27] Ieeju P P, Investigations on some technologically important polymer nunocomposite films and semi crystalline Polypyrrole films, Phd thesis, Cochin University of Science and Technology Cochin - 682 022, Kerala, India, 2012.

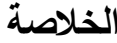

لقد تمت دراسة الصفات البصرية اللاخطية لاغشية الصبغة الليزرية الاوكسازين المطعمة مع بوليمير البولسترين PS و بوليمير اللبوليمثيل اكريليت PMMA و مزيج كل من البوليمرين PS و PMMA تم ترسيب هذه الاغشية بتقنية الطرح الحر و ميزت بتقنية المسح على البعد الثالث. تجربة المسح على البعد الثالث نفذت باستخدام ليزر الدايود ذو الموجات المستمرة وبطول موجي 650 نانومتز و بجزأين. في الجزء الأول تم وضع فتحة أمام الكاثف لغرض دراسة

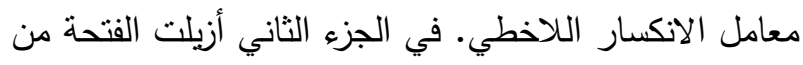
أمام الكاشف (open aperture) لغعرض قياس الأنسار معامل

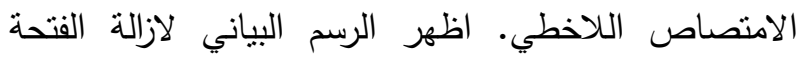

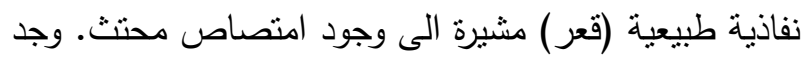
أن اللاخطية الملاحظة هي من المرتبة الثالثة،لانها تتطابق مع عملية إمتصاص فوتونين. أظهر منحني الفتحة المغلقة شكل قمة الى قعر مشيرا الى معامل انكسار لاخطي ذو قيمة

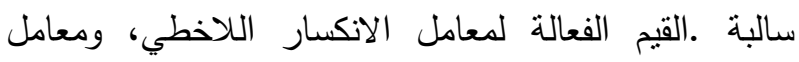

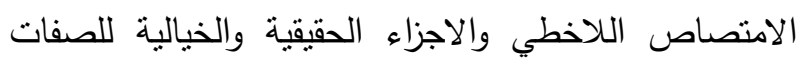
OX/PMMA-PS اللاخطية من المرتبة الثالثة لاغشية مقارنة مع اغشية Ox/PMMA واغشية Ox/PS مع القيمة الواطئة جداً للنفاذية التي تم الحصول عليها اوضحت بإمكانية استخدم هذه الاغشية كمحدات بصرية فعالة. 